\title{
Finite Element Analysis of Stress and Natural Mode for Micro Aero-engine Turbine Disk
}

\author{
Feng Ding ${ }^{1, a^{*}}$, Chuan $\mathrm{Jia}^{2, \mathrm{~b}}$ and Xiang $\mathrm{Li}^{3, \mathrm{C}}$ \\ 1, 2, 3Xi'an Technological University, Xi'an, China \\ adfeng@xatu.edu.cn, bwailxue1999@163.com, xatuxiangli@163.com
}

Keywords:Turbine disk; Stress; Natural mode of vibration; Finite element; Least square method.

\begin{abstract}
In this paper, finite element stress analysis of a micro aero-engine turbine disk with different rotational forces is performed by applying Solidworks and Abaqus. Maximum safe speed of aero-engine is calculated and verificated according to the finite element analysis results. Then maximum stresses in different speeds can be calculated. Natural mode of turbine disk-blade system which is constrained by connecting hole is analyzed, and the first six order inherent frequencies and modal shapes are obtained. Stress analysis provides a theoretical basis for aero-engine speed control, design and optimization of turbine disk. The inherent mode lays the foundation for aero-engine transient analysis, spectral analysis and dynamic analysis.
\end{abstract}

\section{Introduction}

Turbine disk is one of the most critical parts of aero-engine. As the aircraft often work at startup, acceleration, deceleration, constant speed, cut-off and other conditions, engine turbine disk is susceptible to a variety of complex loads, including high-speed operation of the turbine disk by centrifugal force, thermal stress under high temperature, high pressure and other aerodynamic force. In addition, the etching gas also affects the reliability of the turbine disk. The changing operating modes make the turbine disk subjected to alternating load, it will be appear fatigue deformation even cracks under alternating load for a long time. Therefore the turbine disk stress and modal analysis are important.

For finite element analysis on the turbine disk, domestic and foreign scholars have made a lot of research. The thermal elasticity, elastic stress and thermal stress of aero-engine powder metallurgy turbine disk were analyzed by Y.D. Song et al [1] with the finite element method. In literature $[2,3,4]$, they conducted the residual stress distribution simulation by using a finite element model. R. Jiang et al [5] studied the phenomenon of crack in turbine disk alloy by the temperature test, and calculated the fatigue life. R.G. Zhao et al [6] conducted fatigue crack propagation experiment which was under condition of different stress ratios and at room temperature for aero-engine turbine disk made by GH4133B alloy. G.H. Farrahi et al [7] conducted failure analysis using the finite element method for a gas turbine disk, the study found the mechanical fatigue lead to microcracks producing. S.A. Meguid et al [8] considered the influence of the thickness and interfacial friction in the situation of three dimensions. The modal analysis of blade-turbine disk system was conducted by A. Chatterjee, and one of purpose of the research was identify damage based on the modal spectrum [9]. M. Salio et al [10] studied turbine disk residual stress changes under different conditions with MSC Software, and predicted the minimum cutting depth when turning.

Compared with the development of the finite element analysis at home and abroad, the results have obtained certain limitations due to the different scholars adopted different methods and technical means. In finite element analysis, the results are influenced by the error of model and experiment data itself. In the analysis of this paper, relatively precise model is established based on 
the solid size of a micro aero-engine turbine disk, in which defined constraints of connection hole simulating actual situation for more precise. High order polynomial fitting is applied for equivalent compressive stress data via Matlab. In order to guarantee the accuracy of calculation, least square method is adopted to determine the highest polynomial coefficient.

\section{Finite element modeling and stress analysis}

The structure of micro turbojet engine is shown in Fig. 1, and it is supplied by Shanghai Micro Aero Engine Co. Ltd. The finite element simplified model of turbine disk for micro turbojet aero-engine is shown in Fig. 2, which is built in Solidworks. The alloy material (GH4169) is used in finite element analysis for turbine disk and blade. And the section of model is homogeneous elastic solid, 13 generic static analysis is created in the analysis step window. The linear four nodes tetrahedral element is used in model meshing. In the global seed control window, the global approximate size is set to 1.5 and other parameters keep default. After meshing, the total number of nodes is 26337 , and the total number of elements is 108285 . The Fig. 3 shows the loads and restraints in stress analysis.

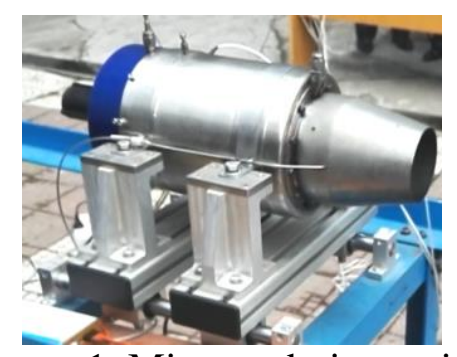

Figure 1. Micro turbojet engine

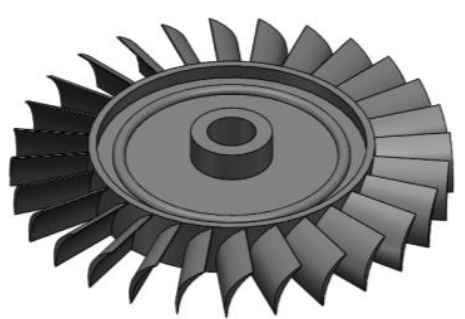

Figure 2. Solid model

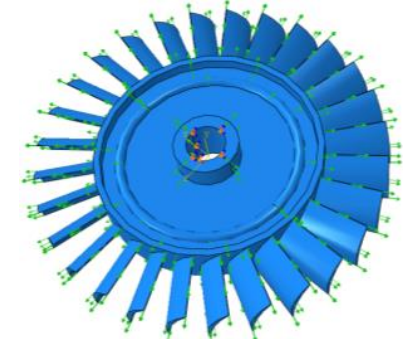

Figure 3. Loads and restraints

A load step is created in the load window and the load type is set to rotational body force. The centrifugal force is applied to the whole model of disk-blade, in which set the Y-axis as a rotation axis. In the window of creating boundary conditions, the displacement of $\mathrm{X}, \mathrm{Y}$ and $\mathrm{Z}$ direction is fixed as well as the rotation of $\mathrm{X}$ and $\mathrm{Y}$ axis for turbine disk as shown as the red dots located in near the connection hole in Fig. 3. The rotational body force is loaded in the entire model. The range of loading angular velocity is $1000-13000 \mathrm{rad} / \mathrm{s}$, and the load interval is $1000 \mathrm{rad} / \mathrm{s}$ as shown as the green arrow portion around the model in Fig. 3.

The Fig. 4 shows the sectional view after deformed and before deformed. In Fig. 4, the part of meshed is undeformed and the others is deformed. The deformation result of the analysis step is shown in Table 1. The top of turbine disk out ring and the out part of connection hole are subjected to a large stress when the turbine disk running in high speed of $8000 \mathrm{rad} / \mathrm{s}$ angular velocity. The deformation of the blade is in the direction of the axial direction and the maximum deformation amount is $0.34 \mathrm{~mm}$.

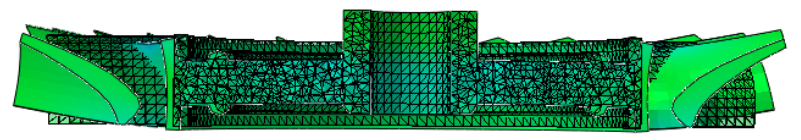

Figure 4. Equivalent compressive stress nephogram

With the increase of load, the analysis results show that the maximum stress value increases. When the angular velocity is $8000 \mathrm{rad} / \mathrm{s}$, the maximum value of equivalent compressive stress is $909.05 \mathrm{MPa}$, and the maximum point is located at the node -1.54906 . Its basic coordinate is $(-10$, $-3.85,-13)$ which located near to the connection hole. The minimum value of equivalent compressive stress is $-742.9 \mathrm{MPa}$. The minimum point is located at the node -1.77216 which located at connection of disk and blade. The values obtained in each analysis step are shown in Table 2. 
Table 1 Turbine deformation results

\begin{tabular}{lcccccc}
\hline Direction & \multicolumn{2}{c}{ X } & \multicolumn{2}{c}{ Y } & \multicolumn{2}{c}{ Z } \\
\hline Max/Min & Max & Min & Max & Min & Max & Min \\
Strain [mm] & $3.3372 \mathrm{e}-1$ & $-3.3209 \mathrm{e}-1$ & $8.8348 \mathrm{e}-2$ & $-3.6242 \mathrm{e}-2$ & $3.3645 \mathrm{e}-1$ & $-3.4118 \mathrm{e}-1$ \\
Node & -1.1731 & -1.1427 & -1.1299 & -1.13866 & -1.3272 & -1.1280 \\
\hline
\end{tabular}

Table 2 Angular velocity-maximum equivalent compressive stress

\begin{tabular}{llllllll}
\hline$\omega[\mathrm{rad} / \mathrm{s}]$ & 1000 & 2000 & 3000 & 4000 & 5000 & 6000 & 7000 \\
\hline$F_{P}[\mathrm{MPa}]$ & 14.20 & 56.82 & 127.84 & 227.26 & 355.10 & 511.34 & 695.99 \\
$\omega[\mathrm{rad} / \mathrm{s}]$ & 8000 & 9000 & 10000 & 11000 & 12000 & 13000 & \\
$F_{P}[\mathrm{MPa}]$ & 909.0 & 1150.5 & 1420.4 & 1718.7 & 2045.4 & 2400.5 & \\
\hline
\end{tabular}

In order to get the relationship of the angular velocity applied on the model and the maximum stress after obtaining the stress data of each analysis, the curve fitting for data points is needed. The polynomial fitting based on least square method and comparative analysis with Matlab programming is adopted to improve the precision of curve fitting. The equivalent stress data processing method is described as following section.

If the data measured by the experiment is $\left(x_{i}, y_{i}\right)(\mathrm{i}=1,2,3, \ldots, \mathrm{m}), \quad \Phi$ is the function class of all times not more than $n(n \leq m)$. In particular, when $n=1$, it is called the linear fitting or the straight line fitting. we need to seek a function $P_{n}=\sum_{k=0}^{n} a_{k} x_{k} \in \Phi$ in order to achieve the equation as follows.

$$
I=\sum_{i=0}^{m}\left[P_{n}\left(x_{i}\right)-y_{i}\right]^{2}=\sum_{i=0}^{m}\left(\sum_{k=0}^{n} a_{k} x_{i}^{k}-y_{i}\right)^{2}=\min
$$

When the highest power of polynomials is 12 , the standard deviation reaches the minimum value. When the engine is working, If the centrifugal force $(F)$ is greater than the strength limit of the material, it causes the failure of the turbine disk. According to the ultimate strength of blade, the angular velocity of polynomial is solved. Angular velocity $\omega_{\text {lim }}=8242.5295 \mathrm{rad} / \mathrm{s}$, namely, the highest safe speed is $n_{\text {lim }}=78712.68 \mathrm{r} / \mathrm{min}$. When applied rotating force load $F_{\text {lim }}$ on model in the finite element analysis software Abaqus once again, the maximum equivalent stress value of analysis is $965.0298 \mathrm{MPa}$ and the solution error is $0.005 \%$, indicating that the curve fitting has higher precision.

The coefficients of the polynomial can be solved by MATLAB program, then we can obtain the fitting function:

$$
\begin{aligned}
& P(x)=-1.59 e-44 x^{12}+1.29 e-39 x^{11}-4.64 e-35 x^{10}+9.78 e-31 x^{9}-1.34 e-26 x^{8}+1.26 e-22 x^{7} \\
& -8.24 e-19 x^{6}+3.78 e-15 x^{5}-1.20 e-11 x^{4}+2.558 e-08 x^{3}-2.01 e-05 x^{2}+0.026 x-973.27
\end{aligned}
$$

In the function, $x$ is the angular velocity of the turbine disk, and the maximum equivalent compressive stress at any velocity can be obtained via the Eq.2.

\section{Natural mode analysis of turbine disk}

The previous model is imported in Abaqus and linear perturbation-frequency is set as the analysis type. Lanczos eigenvalue solver is applied in modal analysis. In order to obtain more modes, the former 20-order intrinsic frequency is obtained. In the step of load, model is only imposed constraints to restrain $\mathrm{X}, \mathrm{Y}, \mathrm{Z}$ direction displacement and $\mathrm{X}, \mathrm{Z}$ direction rotation of turbine disk coupling holes. The remaining steps are similar to previous stress analysis. After the setup is 
completed, job is created and submitted for analysis.

The characteristic frequencies of each order and vibration modes of turbine disk-blade could be viewed in the visualization. Actual object vibration is the superposition effect of all modes. In theory, there are infinite order modes and vibration is superposition of the infinite order modes. Meanwhile the contributions of each mode to system vibration are different. The previous orders are larger, and the later are smaller. It can be seen from the analysis results, there is no obvious vibration mode from the beginning of the seventh order. Therefore the first 6 order modes are the most significant parts to be analyzed. The first six order modal shapes are shown in Fig. 5(a)-(f), characteristic frequencies and modes are shown in Table 3.

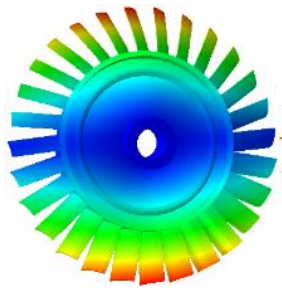

(a)

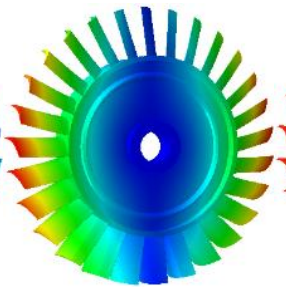

(b)

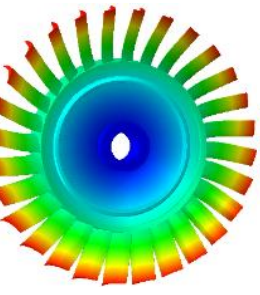

(c)

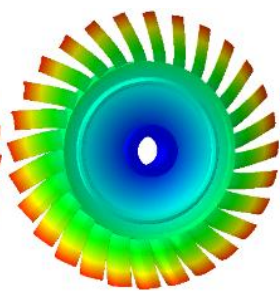

(d)

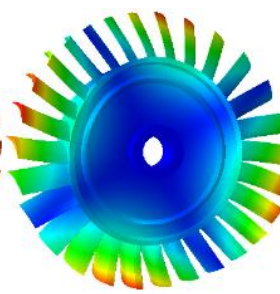

(e)

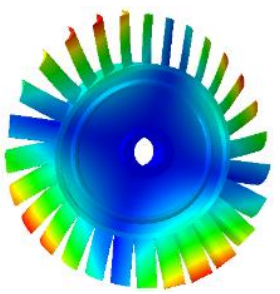

(f)

Figure 5. The first six order modal shapes

Table 3 Characteristic frequency and modes of vibration

\begin{tabular}{cccl}
\hline Modal order & Frequency $[\mathrm{Hz}]$ & Max vibration displaceme $[\mathrm{mm}]$ & Modes of vibration \\
\hline 1,2 & $3654.6,3654.8$ & $1.0384,1.0377$ & 1 nodal diameter \\
3,6 & $4257.5,4843.8$ & $1.2166,1.0337$ & 1 pitch circle \\
4,5 & $4637.8,4638.7$ & $1.0947,1.1191$ & 2 nodal diameter \\
\hline
\end{tabular}

The first order mode shape is described in Fig. 5(a). The pitch diameter is the symmetrical shaft of turbine disk blade, and both sides are made of reverse vibration. Vibration of part which far away symmetry axis is obvious and deformation of top blade which is in the direction of vertical pitch diameter is maximum. Interaction moment between turbine and shaft is formed by both sides reverse vibration, and shaft bending vibration which will destroy the balance of rotor is drived. The cross section is shown in Fig. 6(a). Fig. 5(b) is the second mode shape. It is similar to the first mode, whereas pitch diameter is perpendicular to the first order mode.

Fig. 5(c) is the third mode shape. The shape of the third order mode is as an umbrella, it is called as umbrella vibration. Vibration pitch circle is located in the inside of wheel hub, and vibration is becoming more and more obvious from the connection hole to outer ring. Deformation of top blade which belongs to outer ring is the most evident. Umbrella vibration is surged in axial direction, and the axial stress is emerged which follows circumference of turbine disk. It will cause certain effect to turbine disk support, and the cross section is shown in Fig. 6(b).

The fourth vibration shape is shown in Fig. 5(d), two pitch diameters are orthogonal to each other and their both sides vibrate reversely which also known as fan vibration, cross section is presented in Fig. 6(c). The fan vibration is easy to be caused by rotor imbalance, asymmetric axial force, axial distribution force and material heterogeneity. In spite of the moment which is produced by fan vibration could be offset, a greater force will emerges at the blade root which is located in the middle of the two sections. Fig. 5(e) is the fifth order vibration shape which is same as the fourth mode shape, while pitch diameter deflect a certain angle. Fig. 5(f) is the sixth order modal shape which has a pitch circle, and the main deformation region is distributed between hub to shroud. With the increase of nodal circle radius, deformation becomes more and more obvious, 
among which the deformation amount of top blade is maximum. Deformation direction is mainly circumferential anticlockwise migration.

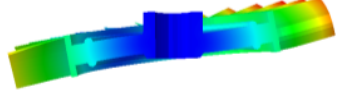

(a) Symmetric vibration

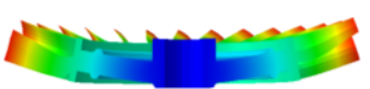

(b) Umbrella vibration

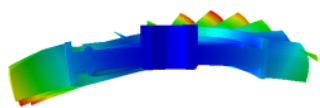

(c) Fan vibration

Figure 6. Three common cross-section of vibration modes

\section{Summary}

(1) In operation of the turbine disk, the outer ring blade is subjected to the axial force and the compressive stress at one side of the blade, and the turbine disk connection hole is subjected to a large tensile stress.

(2) When the maximum number of quadratic polynomial is 12 , the fitting precision is the highest. The standard deviation is $6.1199 \mathrm{e}-09$, and the function expression is obtained. The maximum safe speed of the engine is calculated according to the ultimate strength of the turbine disk materials, which is $78712.68 \mathrm{r} / \mathrm{min}$ and is greater than the maximum speed of the micro engine experiment. Therefor it is in good accordance with the fact.

(3) The first six order mode information of the turbine disk is obtained. The results show the range of frequencies is between 3654.6 to $4843.8 \mathrm{~Hz}$ and the elastic deformation of the different degrees and different vibration mode appears. The umbrella vibration has greatest impact on turbine disk, because the deformation distribution is not balanced and more obvious. Secondly, the fan vibration of two pitch diameter has more influence.

\section{Acknowledgments}

This research is supported by the National Natural Science Foundation of China (No.51275374) and the Fund Project of Science \& Technology on Reliability \& Environmental Engineering Key Laboratory.

\section{References}

[1] Y.D. Song, W. Chen and W.D. Wen: Hangkong Dongli Xuebao, Vol. 12 (1997) No.4, p.422-424, (In Chinese).

[2] U. Cihak, P. Staron and H. Clemens: Materials Science and Engineering: A, Vol. 437 (2006) No.1, p.75-82.

[3] H.Y. Bae, K.J Kim and J.H Kim: International Journal of Pressure Vessels \& Piping, Vol. 114 (2013) No.1, p.1-15.

[4] R.M. Nejad: Engineering Failure Analysis, Vol. 45 (2014) No.1, p.449-455.

[5] R. Jiang, S. Everitt and N. Gao: International Journal of Fatigue, Vol. 75 (2015) No.2, p.89-99.

[6] R.G. Zhao, X.Y. Luo and L.L. Ren: Jixie Gongcheng Xuebao, Vol. 18 (2011) No.47, p.55-65, (In Chinese).

[7] G.H. Farrahi, M. Tirehdast and E.M.K. Abad: Engineering Failure Analysis, Vol. 18 (2010) No.1, p. 474-484.

[8] S.A. Meguid, P.S. Kanth and A. Czekansk: Finite Elements in Analysis \& Design, Vol. 35 (2000) No.4, p.305-317.

[9] A. Chatterjee: Applied Mathematical Modelling, Vol. 40 (2016) No.3, p.2119-2133.

[10]M. Salio, T. Berruti and G.D. Poli: International Journal of Mechanical Sciences, Vol. 48 (2006) No.9, p.976-984. 